Article

\title{
Quantum Quasigroups and the Quantum Yang-Baxter Equation
}

\author{
Jonathan Smith
}

Department of Mathematics, Iowa State University, Ames, IA 50011, USA; jdhsmith@iastate.edu; Tel.: +1-515-294-8172

Academic Editor: Florin Felix Nichita

Received: 11 October 2016; Accepted: 1 November 2016; Published: 9 November 2016

\begin{abstract}
Quantum quasigroups are algebraic structures providing a general self-dual framework for the nonassociative extension of Hopf algebra techniques. They also have one-sided analogues, which are not self-dual. The paper presents a survey of recent work on these structures, showing how they furnish various solutions to the quantum Yang-Baxter equation.
\end{abstract}

Keywords: Hopf algebra; quantum group; quasigroup; Moufang loop; quantum Yang-Baxter equation

AMS: 20N05; 16T25

\section{Introduction}

The self-dual concept of a quantum quasigroup offers a far-reaching unification of Hopf algebras (along with their non-associative extensions, e.g., [1-4]) and quasigroups [5]. Consider a bimagma $(A, \nabla, \Delta)$, an object of a strict symmetric monoidal category $\mathbf{V}$ with $\mathbf{V}$-morphisms giving a magma structure or multiplication $\nabla: A \otimes A \rightarrow A$, and a comagma structure or comultiplication $\Delta: A \rightarrow A \otimes A$, such that $\Delta$ is a magma homomorphism. (The latter bimagma condition is equivalent to its dual: $\nabla$ is a comagma homomorphism.) A bimagma $(A, \nabla, \Delta)$ is a quantum quasigroup if two dual morphisms are invertible in $\mathbf{V}$ : the left composite

$$
\mathrm{G}: A \otimes A \stackrel{\Delta \otimes 1_{A}}{\longrightarrow} A \otimes A \otimes A \stackrel{1_{A} \otimes \nabla}{\longrightarrow} A \otimes A,
$$

("G" for "Gauche") and the right composite

$$
\text { כ: } A \otimes A \stackrel{1_{A} \otimes \Delta}{\longrightarrow} A \otimes A \otimes A \stackrel{\nabla \otimes 1_{A}}{\longrightarrow} A \otimes A,
$$

("כ" for "Droite"). The qualifiers "left" and "right" refer to the side of the tensor product on which the comultiplications appear. Quantum quasigroups also have one-sided analogues [6]. A left quantum quasigroup just requires the invertibility of the left composite. Dually, a right quantum quasigroup requires the invertibility of the right composite.

The present work surveys some connections between these structures and the quantum Yang-Baxter equation (QYBE):

$$
R^{12} R^{13} R^{23}=R^{23} R^{13} R^{12}
$$

[7] (Section 2.2C). The QYBE applies to an endomorphism $R: A \otimes A \rightarrow A \otimes A$ of the tensor square of an object $A$ in a symmetric, monoidal category. For a given integer $n>1$, the notation $R^{i j}$, for $1 \leq i<j \leq n$, means applying $R$ to the $i$-th and $j$-th factors in the $n$-th tensor power of $A$. Since the left and right composite morphisms are endomorphisms of tensor squares, it is natural 
to seek conditions under which they satisfy the QYBE. Then, as observed by B.B. Venkov working in the category of sets with Cartesian products [8] (Section 9), the QYBE corresponds to various distributivity conditions involving magma multiplications $\nabla: A \otimes A \rightarrow A$ appearing in the left and right composites along with diagonal or setlike comultiplications. While such classical distributivity conditions receive due consideration in this survey, the main novelty lies in the combination with more general comultiplications to obtain a much broader palette of QYBE solutions, corresponding to the bialgebraic concept of quantum distributivity as recently introduced in $[9,10]$. It should also be noted (compare Remark 3) that distinct quantum quasigroups may furnish identical solutions to the QYBE. In other words, a classification of quantum quasigroups that exhibit quantum distributivity will be finer than a classification of the QYBE solutions that they provide.

The broad layout of the paper is as follows. The second section mainly covers background material about magmas and quasigroups, including some relations between the classical and quantum concepts of a quasigroup (Section 2.4), and classical concepts of idempotence and distributivity (Section 2.5), including a brief dictionary of the relevant knot theory terminology (Remark 2). The third section introduces the bialgebraic or "quantum" versions of idempotence (Section 3.1), distributivity (Section 3.2), and Albert's notion of isotopy (Section 3.3), in particular showing how quantum distributivity is transferred under bimagma isotopy (Theorem 4). The final section of the paper then presents various QYBE solutions obtained using quantum quasigroup techniques from one of the subtlest structures in quasigroup theory, commutative Moufang loops. While most of the QYBE solutions appearing in the paper are explicitly given in the symmetric monoidal category of sets under the Cartesian product, they may be transferred easily to categories of modules under the tensor product by application of the free module functor-compare [11] (III, Ex. 3.6.3; IV, Proposition 2.4.8(d)).

For concepts and conventions that are not otherwise discussed in this paper, readers are referred to [11]. We use algebraic notation, placing functions to the right of their arguments, or as superfixes. This enables compositions to be read in natural order from left to right. These conventions serve to control the profusion of brackets that all too easily arise in nonassociative structures. Since the comultiplications featured in this paper are not necessarily coassociative, the adapted version

$$
\Delta: A \rightarrow A \otimes A ; a \mapsto a^{L} \otimes a^{R}
$$

of Sweedler notation is used (without any implication that the image of $a$ is of tensor rank 1 ) in concrete symmetric monoidal categories. Note that in this notation, coassociativity takes the form

$$
a^{L L} \otimes a^{L R} \otimes a^{R}=a^{L} \otimes a^{R L} \otimes a^{R R} .
$$

Under coassociativity, the usual Sweedler notation is recovered for example by taking the superfixes $L L<L R<R$ or $L<R L<R R$ in lexicographic order as suffices $1<2<3$ (which are sometimes encased in parentheses).

\section{Magmas, Quasigroups, and Loops}

\subsection{Magmas}

Classically, a magma $(Q, \cdot)$ is a set $Q$ carrying a binary multiplication operation. The operation is generally written as - or otherwise indicated by simple juxtaposition of the two arguments, with · binding less strongly than juxtaposition (The older term groupoid [12] (p. 1) is now best reserved for categories with invertible morphisms). Thus $\nabla: Q \otimes Q \rightarrow Q ; x \otimes y \mapsto x \cdot y$ is a magma in the symmetric, monoidal category of sets under the Cartesian product, writing $x \otimes y$ in place of the usual ordered pair notation $(x, y)$. The magma $\tau \nabla: Q \otimes Q \rightarrow Q ; x \otimes y \mapsto y \cdot x$ is described as the opposite of the magma $(Q, \nabla)$ or $(Q, \cdot)$. Here and elsewhere, the twist morphism of the symmetric, monoidal category in question is written as $\tau: A \otimes A \rightarrow A \otimes A$, for example concretely implemented as $x \otimes y \mapsto y \otimes x$. 
For a set $Q$, the transformation monoid $Q^{Q}$ is the monoid of self-maps from $Q$ to $Q$. Now for an element $q$ of a magma $(Q, *)$, define the left multiplication $L_{*}(q)$ or

$$
L(q): Q \rightarrow Q ; x \mapsto q * x
$$

and right multiplication $R .(q)$ or

$$
R(q): Q \rightarrow Q ; x \mapsto x * q
$$

Specifying

$$
L: Q \rightarrow Q^{Q} ; q \mapsto L(q)
$$

or

$$
R: Q \rightarrow Q^{Q} ; q \mapsto R(q)
$$

is then equivalent (by Currying) to specification of the magma structure. In particular, using the algebraic convention for composition of maps, the associativity of a magma is equivalent to $R$ being a magma homomorphism.

\subsection{Combinatorial and Equational Quasigroups}

Quasigroups may be defined combinatorially or equationally. Combinatorially, a quasigroup $(Q, \cdot)$ is defined as a magma with the property that any two of the arguments $x, y, z$ in the equation $x \cdot y=z$ determine the third uniquely. A loop is defined as a quasigroup $Q$ having an element 1, the identity element, satisfying $1 \cdot x=x=x \cdot 1$ for each $x$ in $Q$.

Under the equational definition, a quasigroup $(Q, \cdot, /, \backslash)$ is a set $Q$ carrying binary operations of multiplication, right division / and left division \, which together satisfy the following identities:

$$
\begin{array}{lll}
\text { (SL) } \quad x \cdot(x \backslash z)=z ; & \text { (SR) } \quad z=(z / x) \cdot x, \\
\text { (IL) } \quad x \backslash(x \cdot z)=z ; & \text { (IR) } \quad z=(z \cdot x) / x .
\end{array}
$$

Note that (SL) gives the surjectivity of the left multiplication $L(x): Q \rightarrow Q$, while (IL) expresses its injectivity. The other two identities are their mirror images (chiral duals).

When an abelian group is considered as a combinatorial quasigroup under subtraction, the addition operation is the right division, while subtraction becomes the left division. If $x$ and $y$ are elements of a group $(Q, \cdot)$, the left division is given by $x \backslash y=x^{-1} y$, with $x / y=x y^{-1}$ as right division. More generally, these equations hold in a diassociative loop, where the subloop generated by each pair of elements is associative, and thus forms a group. The loop $S^{7}$ of unit octonions is diassociative, but not associative [13] (Section 1.7).

\subsection{Equational and Combinatorial One-Sided Quasigroups}

Using an equational definition, a left quasigroup $(Q, \cdot, \backslash)$ is a set $Q$ equipped with a multiplication and left division satisfying the identities (SL) and (IL) of Equation (6). Dually, a right quasigroup $(Q, \cdot, /)$ is a set $Q$ equipped with a multiplication and right division satisfying the identities (SR) and (IR) of Equation (6). A left loop is a left quasigroup with an identity element. Dually, a right loop is a right quasigroup with an identity element.

Combinatorially, a left quasigroup $(Q, \cdot)$ is a set $Q$ with a multiplication such that in the equation $a \cdot x=b$, specification of $a$ and $b$ determines $x$ uniquely. In equational terms, the unique solution is $x=a \backslash b$. The combinatorial definition of right quasigroups is dual. If $Q$ is a set, the right projection product $x y=y$ yields a left quasigroup structure on $Q$, while the left projection product $x y=x$ yields a right quasigroup structure. 
The following algebraic observations are often useful. In particular, they underlie simple conceptual proofs of results (such as Proposition 1 below) that otherwise require more complicated computational or syntactical proofs.

Lemma 1. Let $(P, \cdot)$ and $(Q, \cdot)$ be magmas.

(a) If $(P, \cdot, /)$ is a right quasigroup, then so is $(P, /, \cdot)$.

(b) If $(P, \cdot, \backslash)$ is a left quasigroup, then so is $(P, \backslash, \cdot)$.

(c) If $(P, \cdot, /)$ and $(Q, \cdot, /)$ are right quasigroups, and $f: P \rightarrow Q$ is a magma homomorphism, then it is a right quasigroup homomorphism.

(d) If $(P, \cdot, \backslash)$ and $(Q, \cdot, \backslash)$ are left quasigroups, and $f: P \rightarrow Q$ is a magma homomorphism, then it is a left quasigroup homomorphism.

Proof. Claims (a) and (b) are immediate from the definitions. Claim (c) will be proved and the claim (d) is dual. For elements $x$ and $z$ of $P$, one has $z=(z / x) \cdot x$ by (SR). Thus, $z^{f}=(z / x)^{f} \cdot x^{f}$, whence $z^{f} / x^{f}=\left[(z / x)^{f} \cdot x^{f}\right] / x^{f}=(z / x)^{f}$ by (IR).

\subsection{Quasigroups and Quantum Quasigroups}

This paragraph summarizes some connections between quasigroups and quantum quasigroups. The symbol $T$ is often used to denote a singleton set, in its role as a terminal object of categories of sets and functions.

Recall that a unital magma $(A, \nabla, \eta)$ in a symmetric, monoidal category $(\mathbf{V}, \otimes, \mathbf{1})$ is a magma $(A, \nabla)$ equipped with a unit $\mathbf{V}$-morphism $\eta: \mathbf{1} \rightarrow A$ such that $\left(1_{A} \otimes \eta\right) \nabla=1_{A}=\left(\eta \otimes 1_{A}\right) \nabla$. Dually, a counital comagma $(A, \Delta, \varepsilon)$ within a symmetric, monoidal category $(\mathbf{V}, \otimes, \mathbf{1})$ is a comagma $(A, \Delta)$ equipped with a counit $\mathbf{V}$-morphism $\varepsilon: A \rightarrow \mathbf{1}$ such that $\Delta\left(1_{A} \otimes \varepsilon\right)=1_{A}=\Delta\left(\varepsilon \otimes 1_{A}\right)$. In particular, in the symmetric, monoidal category $($ Set,$\times, \top$ ) of sets with Cartesian products, the only counital comultiplication is the diagonal or setlike comultiplication $\Delta: A \rightarrow A \otimes A ; x \mapsto x \otimes x$ [10] (Lemma 4.6).

Theorem 1. [5,6] Consider the category Set of sets and functions, with the symmetric monoidal category structure $(\mathbf{S e t}, \times, \top)$. Then, counital left, right, or two-sided quantum quasigroups in (Set, $\times, \top)$ are, respectively, equivalent to left, right, or two-sided quasigroups.

Theorem 2. Consider the symmetric, monoidal category (FinSet, $x, T$ ) of finite sets under the Cartesian product:

(a) Left quantum quasigroups in (FinSet, $\times, T$ ) are equivalent to triples $(A, L, R)$ that consist of a left quasigroup $A$ with an automorphism $L$ and endomorphism $R$ [6].

(b) Right quantum quasigroups in (FinSet, $\times, T)$ are equivalent to triples $(A, L, R)$ that consist of a right quasigroup $A$ with an endomorphism $L$ and automorphism $R$ [6].

(c) Quantum quasigroups in (FinSet, $\times, T$ ) are equivalent to triples $(A, L, R)$ consisting of a quasigroup $A$ equipped with automorphisms $L$ and $R$ [5].

Corollary 1. [6] Given a left quasigroup $(A, \cdot, \backslash)$ equipped with an automorphism $L$ and endomorphism $R$, define $\nabla: A \otimes A \rightarrow A ; a \otimes b \mapsto$ ab as a multiplication and $\Delta: A \rightarrow A \otimes A ; a \mapsto a^{L} \otimes a^{R}$ as a comultiplication. Then, $(A, \nabla, \Delta)$ is a left quantum quasigroup in $(\mathbf{S e t}, \times, \top)$.

The chiral dual is as follows.

Corollary 2. Given a right quasigroup $(A, \cdot, /)$ equipped with an endomorphism $L$ and automorphism $R$, define $\nabla: A \otimes A \rightarrow A ; a \otimes b \mapsto a b$ as a multiplication and $\Delta: A \rightarrow A \otimes A ; a \mapsto a^{L} \otimes a^{R}$ as a comultiplication. Then, $(A, \nabla, \Delta)$ is a right quantum quasigroup in (Set, $\times, \top)$. 
Corollary 3. [5] Suppose that $(A, \cdot, /, \backslash)$ is a quasigroup equipped with two automorphisms $L$ and $R$. Define $\nabla: A \otimes A \rightarrow A ; a \otimes b \mapsto a b$ as a multiplication and $\Delta: A \rightarrow A \otimes A ; a \mapsto a^{L} \otimes a^{R}$ as a comultiplication. Then, $(A, \nabla, \Delta)$ is a quantum quasigroup in (Set, $\times, \top)$.

\subsection{Some Classical Concepts}

Definition 1. Let $(Q, \cdot)$ be a magma:

(a) The magma is idempotent if $x x=x$ for all $x$ in $Q$.

(b) The magma is left repetitive if $x y=(x x) y$ for all $x, y$ in $Q$, i.e., if the monogenic submagmas of $Q$ are contained entirely within kernel classes of the map L of Equation (4).

(c) The magma is right repetitive if $y x=y(x x)$ for all $x, y$ in $Q$, i.e., if the monogenic submagmas of $Q$ are contained entirely within kernel classes of the map $R$ of Equation (5).

(d) The magma is left (self-)distributive if $x(y z)=(x y)(x z)$ for all $x, y, z$ in $Q$, i.e., if each left multiplication Equation (2) is a magma endomorphism.

(e) The magma is right (self-)distributive if $(z y) x=(z x)(y x)$ for all $x, y, z$ in $Q$, i.e., if each right multiplication Equation (3) is a magma endomorphism.

(f) The magma is (self-)distributive if it is right and left distributive.

Remark 1. As observed at the end of Section 2.1, the "global" magma homomorphism property for the map $R$ of Equation (5) corresponds to associativity of the magma in question. On the other hand, the "local" magma homomorphism property for each individual value $R(x)$ corresponds to right distributivity as in Definition 1e.

Remark 2. A quandle is just an idempotent, right distributive right quasigroup (or dually, an idempotent, left distributive left quasigroup) [14] (Definition 14). A rack is a right distributive right quasigroup (or dually, a left distributive left quasigroup) [14] (Definition 27). Racks may also be described by giving the opposite of the division along with the multiplication (as in Proposition 1 below). Crans has used the term shelf for a one-sided distributive magma [15] (p. 52), the term spindle for a one-sided idempotent distributive magma [15] (p. 53), and the term quasi-idempotent for repetitive magmas [15] (Section 3.1.3).

Proposition 1. Let $(Q, \cdot, /)$ be a right quasigroup, with opposite division $x \% y=y / x$ :

(a) If the magma $(Q, \cdot)$ is right distributive, then so is the magma $(Q, /)$.

(b) If the magma $(Q, \cdot)$ is right distributive, then the magma $(Q, \%)$ is left distributive.

(c) If the magma $(Q, \cdot)$ is right distributive, then it is right repetitive.

Proof. (a): If the magma $(Q, \cdot)$ is right distributive, its right multiplications and their inverses are magma automorphisms. Then, by Lemma $1(\mathrm{c})$, the right multiplications $R /(q)=R .(q)^{-1}$ are magma automorphisms of $(Q, /)$.

(b): By (a), the magma $(Q, /)$ is right distributive. Thus, its opposite $(Q, \%)$ is left distributive.

(c): For elements $x, y$ of $Q$, one has

$$
(y \cdot x x) / x=(y / x) \cdot[(x x) / x]=(y / x) \cdot x=y,
$$

since $R(x)^{-1}$ is an automorphism of $(Q, \cdot)$. Right multiplication of the extremes of Equation (7) by $x$ then yields the right repetitiveness.

\section{Quantum Idempotence, Distributivity, and Isotopy}

Idempotence, (self-)distributivity, and isotopy are standard concepts for magmas in the monoidal category of sets and Cartesian products. It is convenient to describe them as classical concepts (compare the heading of Section 2.5), as their extensions to quantum concepts in bimagmas provide key tools for the identification of solutions to the QYBE in one- or two-sided quantum quasigroups. 


\subsection{Quantum Idempotence}

For the following definition, compare $[10,16]$.

Definition 2. Let $(A, \nabla, \Delta)$ be a bimagma in a symmetric, monoidal category $\mathbf{V}$. If the diagram

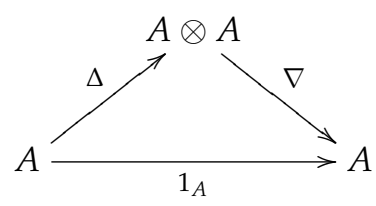

commutes in $\mathbf{V}$, then the bimagma is said to satisfy the condition of quantum idempotence.

A comagma $(A, \Delta)$ is said to be cocommutative if $\tau \Delta=\Delta$. The first result relates classical idempotence to quantum idempotence.

Proposition 2. [10] (Proposition 5.2) Let $(A, \nabla)$ be a magma in (Set, $\times, \top$ ). Define $\Delta: A \rightarrow A \otimes A$; $a \mapsto a \otimes a:$

(a) The structure $(A, \nabla, \Delta)$ is a counital, cocommutative, coassociative bimagma.

(b) The bimagma $(A, \nabla, \Delta)$ is quantum idempotent if and only if the magma $(A, \nabla)$ is idempotent in the classical sense.

Non-classical examples of quantum idempotence are obtained as follows (Compare [10] (Theorem 5.4) for the dual.).

Theorem 3. Let $(A, \cdot, /)$ be a right quasigroup in which the identity

$$
(x / y) \cdot(x / y)=(x \cdot x) /(y \cdot y)
$$

is satisfied. Define

$$
\Delta_{2,1}: A \rightarrow A \otimes A ; a \mapsto a \cdot a \otimes a
$$

and $\nabla: a \otimes b \mapsto a / b$.

(a) The structure $\left(A, \nabla, \Delta_{2,1}\right)$ forms a right quantum quasigroup within the category (Set, $\times, \top$ ).

(b) The bimagma $\left(A, \nabla, \Delta_{2,1}\right)$ is quantum idempotent.

Corollary 4. [10] (Corollary 5.5) Under the conditions of Theorem 3, the bimagma $\left(A, \nabla, \Delta_{2,1}\right)$ is cocommutative if and only if $(A, \nabla)$ is classically idempotent.

Commutative, diassociative loops (such as abelian groups) satisfy the conditions of Theorem 3 , along with entropic right quasigroups (i.e., where the multiplication is a homomorphism), including sets equipped with left projections. The comultiplication (9) of Theorem 3 is also useful in other contexts.

Proposition 3. Let $(A, \cdot)$ be a right distributive right quasigroup. Then, $\nabla: a \otimes b \mapsto a b$ and Equation (9) yield a right quantum quasigroup $\left(A, \nabla, \Delta_{2,1}\right)$.

Proof. The bimagma condition for $\left(A, \nabla, \Delta_{2,1}\right)$ amounts to satisfaction of $(y x)(y x)=(y y)(x x)$ in $(A, \cdot)$. It follows from the right distributivity and right repetitiveness as $(y x)(y x)=(y y) x=(y y)(x x)$, and thus holds by Proposition 1c. Let $\partial_{2}$ be the right composite of $\left(A, \nabla, \Delta_{2,1}\right)$, and let $\partial_{1}$ be the right composite of $(A, \nabla, \Delta)$ with the diagonal comultiplication $\Delta$. Since $\partial_{2}=\partial_{1}$, Corollary 2 shows that $\left(A, \nabla, \Delta_{2,1}\right)$ is a right quantum quasigroup. 


\subsection{Quantum Distributivity}

Definition 3. Suppose that $(A, \nabla, \Delta)$ is a bimagma in a symmetric, monoidal category:

(a) The bimagma $(A, \nabla, \Delta)$ is said to satisfy the condition of quantum left distributivity if the left composite $\mathrm{G}$ of $(A, \nabla, \Delta)$ satisfies the quantum Yang-Baxter equation (1).

(b) The bimagma $(A, \nabla, \Delta)$ is said to satisfy the condition of quantum right distributivity if the right composite D of $(A, \nabla, \Delta)$ satisfies the quantum Yang-Baxter equation (1).

(c) The bimagma $(A, \nabla, \Delta)$ is said to satisfy the condition of quantum distributivity if it has both the left and right quantum distributivity properties.

The following result records B.B. Venkov's motivating observation [8] (Section 9) quoted in the introduction.

Proposition 4. [10] (Proposition 6.2) Let $(A, \nabla)$ be a magma in the category of sets with the Cartesian product. Define $\Delta: A \rightarrow A \otimes A ; a \mapsto a \otimes a$ :

(a) The bimagma $(A, \nabla, \Delta)$ is quantum left distributive if and only if the magma $(A, \nabla)$ is left distributive.

(b) The bimagma $(A, \nabla, \Delta)$ is quantum right distributive if and only if the magma $(A, \nabla)$ is right distributive.

Remark 3. It is noteworthy that the distinct right quantum quasigroups $\left(A, \nabla, \Delta_{2,1}\right)$ and $(A, \nabla, \Delta)$ appearing in the proof of Proposition 3 have identical right composites. By Proposition 4, this common right composite solves the QYBE. Thus, a classification of quantum right distributive right quantum quasigroups would be finer than a classification of the QYBE solutions that they provide.

Corollary 5. [10] (Corollary 6.3) Suppose that $(A, \nabla, \Delta)$ is a nontrivial left quantum distributive left quantum quasigroup, or right quantum distributive right quantum quasigroup, within the category (Set, $\times, \top)$ :

(a) If $(A, \nabla, \Delta)$ is unital, it is not counital.

(b) If $(A, \nabla, \Delta)$ is counital, it is not unital.

Proposition 5. [9] (Proposition 4.4) Let $(A, \nabla, \Delta)$ be a bimagma in (Set, $\times, \top$ ), equipped with comultiplication $\Delta: A \rightarrow A \otimes A ; a \mapsto a^{L} \otimes a^{R}$.

(a) The bimagma $(A, \nabla, \Delta)$ is quantum left distributive if $L R=R L$ and the identity

$$
x^{R}\left(y^{R} z\right)=\left(x^{R R} y^{R}\right)\left(x^{R L} z\right)
$$

is satisfied.

(b) The bimagma $(A, \nabla, \Delta)$ is quantum right distributive if $L R=R L$ and the identity

$$
\left(z y^{L}\right) x^{L}=\left(z x^{L R}\right)\left(y^{L} x^{L L}\right)
$$

is satisfied.

Corollary 6. Let $(A, \nabla, \Delta)$ be a bimagma in (Set, $\times, \top$ ), equipped with comultiplication $\Delta: A \rightarrow A \otimes A$; $a \mapsto a^{L} \otimes a^{R}$.

(a) The bimagma $(A, \nabla, \Delta)$ is quantum left distributive if $L R=R L$ and the identity

$$
x(y z)=\left(x^{R} y\right)\left(x^{L} z\right)
$$

is satisfied [10] (Corollary 6.5). 
(b) The bimagma $(A, \nabla, \Delta)$ is quantum right distributive if $L R=R L$ and the identity

$$
(z y) x=\left(z x^{R}\right)\left(y x^{L}\right)
$$

is satisfied.

3.3. Quantum Homotopy and Isotopy

To begin, recall some classical definitions.

Definition 4. Consider magma structures

$$
\nabla_{i}: A_{i} \otimes A_{i} \rightarrow A_{i} ; a \otimes b \mapsto a \circ_{i} b
$$

(for $i=1,2)$ in a concrete symmetric monoidal category $\mathbf{V}$.

(a) A homotopy (in the classical sense)

$$
(f, g, h):\left(A_{1}, \nabla_{1}\right) \leadsto\left(A_{2}, \nabla_{2}\right)
$$

is defined as a triple of $\mathbf{V}$-morphisms $f, g, h: A_{1} \rightarrow A_{2}$ such that the diagram

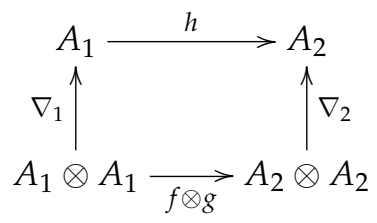

commutes. (See [13] (Section 1.2) for the case of quasigroups in the category of sets.)

(b) The classical homotopy (14) is an isotopy (in the classical sense) if $f, g$, $h$ are $\mathbf{V}$-isomorphisms. (See [17] (Section 11) for the concept in a category of vector spaces, and [18] (Section 4) for quasigroups in the category of sets.)

(c) A classical isotopy $(f, g, h):\left(A_{1}, \nabla_{1}\right) \leadsto\left(A_{2}, \nabla_{2}\right)$ is principal when $A_{1}=A_{2}$ and $h=1_{A_{1}}$.

Remark 4. Note that a classical homotopy (14) becomes a magma homomorphism if $f=g=h$.

Definition 5. Let $(\mathbf{V}, \otimes, \mathbf{1})$ be a symmetric monoidal category. Let $\left(A_{i}, \nabla_{i}\right)$ be a magma in $(\mathbf{V}, \otimes, \mathbf{1})$, for $i=1,2$. Consider a pair $(h, \phi)$ consisting of $\mathbf{V}$-morphisms $h: A_{1} \rightarrow A_{2}$ and $\phi: A_{1} \otimes A_{1} \rightarrow A_{2} \otimes A_{2}$ :

(a) The pair $(h, \phi)$ is a magma homotopy

$$
(h, \phi):\left(A_{1}, \nabla_{1}\right) \leadsto\left(A_{2}, \nabla_{2}\right)
$$

in $(\mathbf{V}, \otimes, \mathbf{1})$ if the diagram

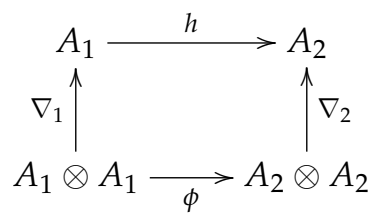

commutes in $\mathbf{V}$.

(b) The magma homotopy (16) is said to be a magma isotopy if h and $\phi$ are $\mathbf{V}$-isomorphisms. 
(c) A magma isotopy (16) is principal when $A_{1}=A_{2}$ and $h=1_{A_{1}}$. Thus, a principal magma isotopy $\left(1_{A_{1}}, \phi\right)$ is specified by its second component $\phi$.

Example 1. For a commutative ring $K$, take the symmetric monoidal category $(\underline{\underline{K}}, \otimes, K)$ of $K$-modules:

(a) Let $(A, \nabla, \eta, \Delta, \varepsilon, S)$ be a Hopf algebra in $(\underline{\underline{K}}, \otimes, K)$. Then,

$$
(S,(S \otimes S) \tau):(A, \nabla) \leadsto(A, \nabla)
$$

is a non-classical magma homotopy.

(b) Let $\left(K_{r}^{r}, \nabla\right)$ denote the algebra of $(r \times r)$-matrices, considered as a magma in $(\underline{\underline{K}}, \otimes, K)$. Let $T: K_{r}^{r} \rightarrow K_{r}^{r}$ denote matrix transposition. Then,

$$
(T,(T \otimes T) \tau):\left(K_{r}^{r}, \nabla\right) \leadsto\left(K_{r}^{r}, \nabla\right)
$$

is a non-classical magma isotopy.

Remark 5. (a) The concepts of Definition 4 provide instances of those of Definition 5.

(b) Under magma homotopies, the class of magmas in $(\mathbf{V}, \otimes, \mathbf{1})$ forms the object class of a category, the homotopy category of magmas in $(\mathbf{V}, \otimes, \mathbf{1})$. The magma isotopies are the isomorphisms in this category.

Definition 6. Consider a symmetric monoidal category $(\mathbf{V}, \otimes, \mathbf{1})$. Let $\left(A, \nabla_{i}, \Delta_{i}\right)$ be a bimagma in $(\mathbf{V}, \otimes, \mathbf{1})$, for $i=1,2$. Then, an automorphism $\phi$ of the object $A \otimes A$ of $\mathbf{V}$ is a principal bimagma isotopy

$$
\phi:\left(A, \nabla_{1}, \Delta_{1}\right) \leadsto\left(A, \nabla_{2}, \Delta_{2}\right)
$$

in $(\mathbf{V}, \otimes, \mathbf{1})$ if the decagon diagram

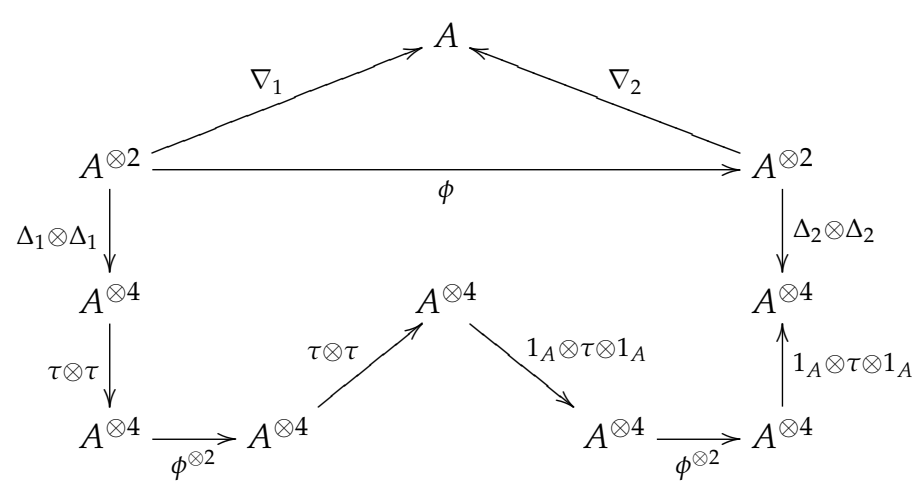

commutes in $\mathbf{V}$. Here, the superfix $\otimes r$ is used for the $r$-th tensor power of an object or morphism in $\mathbf{V}$. The lower part of the decagon diagram is called the nonagon diagram or enneagon diagram.

Remark 6. The commuting of the upper triangle within the decagon diagram means that the pair $\left(1_{A}, \phi\right)$ is a principal magma isotopy.

For the following, compare [9] (Proposition 5.5), which used a dual convention for labeling the domain and codomain of an isotopy.

Proposition 6. Suppose that $\left(Q, \nabla_{i}, \Delta_{i}: x \mapsto x L_{i} \otimes x R_{i}\right)$, for $i=1,2$, are bimagmas in (Set, $\times, \top$ ), with permutations $f: Q \rightarrow Q$ and $g: Q \rightarrow Q$. Then, the validity of the equations

$$
L_{1} g f=f L_{2}, \quad L_{1} g^{2}=g L_{2}, \quad R_{1} f^{2}=f R_{2}, \quad R_{1} f g=g R_{2}
$$


is equivalent to the commuting of the enneagon diagram (18) for a principal bimagma isotopy

$$
f \otimes g:\left(Q, \nabla_{1}, \Delta_{1}\right) \leadsto\left(Q, \nabla_{2}, \Delta_{2}\right)
$$

composed from $f: Q \rightarrow Q$ and $g: Q \rightarrow Q$.

Corollary 7. Consider a magma $\left(Q, \nabla_{2}: x \otimes y \mapsto x \cdot y\right)$ in (Set, $\left.\times, \top\right)$, with commuting automorphisms $f$ and $g$ :

(a) There is a bimagma

$$
\left(Q, \nabla_{2}: x \otimes y \mapsto x \cdot y, \Delta_{2}: x \mapsto x \otimes x\right)
$$

in (Set, $\times, \top)$.

(b) There is a bimagma

$$
\left(Q, \nabla_{1}: x \otimes y \mapsto x f \cdot y g, \Delta_{1}: x \mapsto x g^{-1} \otimes x f^{-1}\right)
$$

in (Set, $\times, \top$ ).

(c) There is a principal bimagma isotopy

$$
f \otimes g:\left(Q, \nabla_{1}, \Delta_{1}\right) \leadsto\left(Q, \nabla_{2}, \Delta_{2}\right) .
$$

Remark 7. In Corollary $7 b$, duality interchanges and inverts the respective components $f, g$ of the magma isotopy to yield the corresponding components $g^{-1}, f^{-1}$ of the comultiplication.

\subsection{Isotopy of Quantum Distributive Structures}

In this section, some results from [9] (Section 6) concerning the preservation of quantum distributivity under principal isotopy are summarized.

Proposition 7. Suppose that $\left(Q, \nabla_{i}: x \otimes y \mapsto x \circ_{i} y, \Delta_{i}: x \mapsto x^{L_{i}} \otimes x^{R_{i}}\right)$ are bimagmas on an object $Q$ of (Set, $\times, \top$ ) for $i=1,2$, such that the following conditions are satisfied:

(a) The comultiplication component $R_{2}: Q \rightarrow Q$ is surjective;

(b) The comultiplication components $L_{i}$ and $R_{i}$ commute for $i=1,2$;

(c) The bimagma $\left(Q, \nabla_{2}, \Delta_{2}\right)$ is quantum left distributive;

(d) There is a principal magma isotopy

$$
f \otimes g:\left(Q, \nabla_{1}\right) \leadsto\left(Q, \nabla_{2}\right)
$$

whose components $f, g$ are commuting automorphisms of $\left(Q, \nabla_{2}\right)$;

(e) The equations $L_{1} g f=f L_{2}$ and $R_{1} f^{2}=f R_{2}$ hold.

Then, $\left(Q, \nabla_{1}, \Delta_{1}\right)$ is quantum left distributive.

The chiral dual of Proposition 7 is formulated as follows.

Corollary 8. Suppose that $\left(Q, \nabla_{i}: x \otimes y \mapsto x \circ_{i} y, \Delta_{i}: x \mapsto x^{L_{i}} \otimes x^{R_{i}}\right)$ are bimagmas on an object $Q$ of (Set, $\times, \top$ ) for $i=1,2$, such that the following conditions are satisfied:

(a) The comultiplication component $L_{2}: Q \rightarrow Q$ is surjective;

(b) The comultiplication components $L_{i}$ and $R_{i}$ commute for $i=1,2$;

(c) The bimagma $\left(Q, \nabla_{2}, \Delta_{2}\right)$ is quantum right distributive; 
(d) There is a principal magma isotopy

$$
f \otimes g:\left(Q, \nabla_{1}\right) \leadsto\left(Q, \nabla_{2}\right)
$$

whose components $f, g$ are commuting automorphisms of $\left(Q, \nabla_{2}\right)$;

(e) The equations $L_{1} g^{2}=g L_{2}$ and $R_{1} f g=g R_{2}$ hold.

Then, $\left(Q, \nabla_{1}, \Delta_{1}\right)$ is quantum right distributive.

The conditions of Proposition 7 and Corollary 8 are simplified and unified for quantum quasigroups in the symmetric monoidal category of finite sets and Cartesian products.

Theorem 4. Suppose that $\left(Q, \nabla_{i}: x \otimes y \mapsto x \circ_{i} y, \Delta_{i}: x \mapsto x^{L_{i}} \otimes x^{R_{i}}\right)$ are quantum quasigroups on an object $Q$ of (FinSet, $\times, \top)$ for $i=1,2$, such that the following conditions are satisfied:

(a) The comultiplication components $L_{i}$ and $R_{i}$ commute for $i=1,2$;

(b) The quantum quasigroup $\left(Q, \nabla_{2}, \Delta_{2}\right)$ is quantum distributive;

(c) There is a principal bimagma isotopy

$$
f \otimes g:\left(Q, \nabla_{1}, \Delta_{1}\right) \sim\left(Q, \nabla_{2}, \Delta_{2}\right)
$$

whose components $f, g$ are commuting automorphisms of $\left(Q, \nabla_{1}\right)$.

Then, $\left(Q, \nabla_{1}, \Delta_{1}\right)$ is quantum distributive.

\section{Commutative Moufang Loops}

\subsection{Moufang Loops}

Proposition 8. [11] (I Proposition 4.1.5) In a loop $(A, \cdot, /, \backslash, 1)$, the following three identities are equivalent:

$$
(z y \cdot z) x=z(y \cdot z x) ; \quad z x \cdot y z=(z \cdot x y) z ; \quad x(z \cdot y z)=(x z \cdot y) z
$$

Definition 7. (a) The identities (20) of Proposition 8 are known as the Moufang identities.

(b) A loop satisfying the Moufang identities is known as a Moufang loop.

Groups are Moufang loops. By Moufang's Theorem [12] (Section VII.4), Moufang loops are diassociative. The loop $S^{7}$ of unit octonions is a non-commutative, non-associative Moufang loop. Following Zassenhaus, Bruck constructed the free commutative Moufang loop on three generators by equipping the abelian group

$$
\mathbb{Z}^{3} \oplus(\mathbb{Z} / 3 \mathbb{Z})=\left\{\mathbf{x}=\left(x_{1}, x_{2}, x_{3}, x_{4}\right) \mid x_{1}, x_{2}, x_{3} \in \mathbb{Z}, x_{4} \in \mathbb{Z} / 3 \mathbb{Z}\right\}
$$

with a non-associative product $\mathbf{x} \cdot \mathbf{y}$ defined as

$$
\mathbf{x}+\mathbf{y}+\left(0,0,0,\left(x_{3}-y_{3}\right)\left(x_{1} y_{2}-x_{2} y_{1}\right)+3 \mathbb{Z}\right)
$$

A representation-theoretic interpretation of this construction is given in [13] (Theorem 11.6). Another powerful method for constructing commutative Moufang loops is discussed in [19].

Proposition 9. Let $(A, \cdot, /, \backslash, 1)$ be a commutative Moufang loop. Define $\Delta: A \rightarrow A \otimes A ; a \mapsto a^{-1} \otimes a^{-1}$ and $\nabla: a \otimes b \mapsto a \cdot b$. In the category (Set, $\times, \top$ ), the structure $(A, \nabla, \Delta)$ forms a unital, commutative and cocommutative quantum quasigroup.

Proof. By the commutativity and diassociativity of $(A, \cdot, /, \backslash, 1)$, the inversion mapping $A \rightarrow A$; $a \mapsto a^{-1}$ is an automorphism of the multiplication $\nabla$. By Corollary 3 , it follows that $(A, \nabla, \Delta)$ is a quantum quasigroup in $($ Set, $\times, \top)$. The remaining statements are immediate. 
Remark 8. Consider the context of Proposition 9:

(a) If $A$ is nontrivial, say with non-identity element a, then

$$
a \Delta\left(\Delta \otimes 1_{A}\right)=\left(a^{-1} \otimes a^{-1}\right)\left(\Delta \otimes 1_{A}\right)=a \otimes a \otimes a^{-1}
$$

while

$$
a \Delta\left(1_{A} \otimes \Delta\right)=\left(a^{-1} \otimes a^{-1}\right)\left(1_{A} \otimes \Delta\right)=a^{-1} \otimes a \otimes a,
$$

so that $(A, \nabla, \Delta)$ is not coassociative.

(b) The quantum quasigroup $(A, \nabla, \Delta)$ is associative if and only if the loop $(A, \cdot, /, \backslash, 1)$ is associative.

(c) The quantum quasigroup $(A, \nabla, \Delta)$ is always unital.

\subsection{Commutative Moufang Loops of Exponent 3}

Since Moufang loops are diassociative, one may define their exponents as for groups. (Generally, definition of the exponent for an individual quasigroup, or a class of quasigroups, requires the use of representation theory [13] (Section 11.2).) The following result shows how commutative Moufang loops of exponent 3 yield QYBE solutions when paired with an appropriate comultiplication.

Theorem 5. [10] (Theorem 6.6) Suppose that $(A, \cdot, /, \backslash, 1)$ is a commutative Moufang loop of exponent 3. Define $\Delta: A \rightarrow A \otimes A ; a \mapsto a^{-1} \otimes a^{-1}$ and $\nabla: a \otimes b \mapsto a \cdot b$. Consider the unital, commutative and cocommutative quantum quasigroup $(A, \nabla, \Delta)$ given by Proposition 9:

(a) The quantum quasigroup $(A, \nabla, \Delta)$ is quantum idempotent.

(b) The quantum quasigroup $(A, \nabla, \Delta)$ is quantum distributive.

Remark 9. In the context of Theorem 5, Proposition 5 shows that the quantum quasigroup $(A, \nabla, \Delta)$ is counital only when $A$ is trivial.

Remark 10. Manin actually takes the identity $x^{2}(y z)=(x y)(x z)$ as a defining axiom for commutative Moufang loops, within the class of loops [20] (I.1.4(4)) (cf. [21] (Theorem II.7B)). Substituting $x^{-1}$ for $x$ and using the exponent 3 condition $\left(x^{-1}\right)^{2}=x$ then directly produces the equation

$$
x(y z)=\left(x^{-1} y\right)\left(x^{-1} z\right)
$$

that interprets the identity (12) of Corollary 6 for the left quantum distributivity of the quantum quasigroup $(A, \nabla, \Delta)$ of Theorem 5 .

\subsection{Belousov's Theorem and Quantum Distributivity}

The classical Belousov Theorem states that for each element $e$ of a distributive quasigroup $\left(Q, \nabla_{2}\right)$, there is an isotopic commutative Moufang loop

$$
\left(Q, \nabla_{1}: x \otimes y \mapsto\left(x R(e)^{-1} \otimes y L(e)^{-1}\right) \nabla_{2}, \eta: 1 \mapsto e\right)
$$

[22] (Teorema 1), [23] (Teorema 8.1). This section (summarizing [9] (Section 7)) shows how Belousov's Theorem may be used in the context of quantum quasigroups to obtain new solutions of the QYBE.

Lemma 2. Let e be an element of a distributive quasigroup $(Q, \cdot)$ :

(a) The left and right multiplications $L(e)$ and $R(e)$ are automorphisms of $(Q, \cdot)$ [23] (p. 131);

(b) The element $e$ is idempotent: $e e=e[23](p .131)$;

(c) The multiplications $L(e)$ and $R(e)$ commute [23] (8.3). 
Theorem 6. Suppose that $\left(Q, \nabla_{2}\right)$ is a distributive quasigroup with an element e. Set $f=R(e)^{-1}$ and $g=L(e)^{-1}$. Let $\left(Q, \nabla_{1}\right)$ be the commutative Moufang loop with multiplication

$$
\nabla_{1}: Q \otimes Q \rightarrow Q ; x \otimes y \mapsto(x f \otimes y g) \nabla_{2}
$$

given by Belousov's Theorem. Then, with the comultiplication

$$
\Delta_{1}: Q \rightarrow Q \otimes Q ; x \mapsto x^{L(e)} \otimes x^{R(e)},
$$

there is a quantum distributive quantum quasigroup $\left(Q, \nabla_{1}, \Delta_{1}\right)$ in the symmetric monoidal category $($ Set, $\times, \top)$.

Corollary 9. In the context of Theorem 6 , with the comultiplication

$$
\Delta_{2}: Q \rightarrow Q \otimes Q ; x \mapsto x \otimes x
$$

Corollary 7 yields a principal bimagma isotopy

$$
f \otimes g:\left(Q, \nabla_{1}, \Delta_{1}\right) \leadsto\left(Q, \nabla_{2}, \Delta_{2}\right) .
$$

Remark 11. (a) If the distributive quasigroup $\left(Q, \nabla_{2}\right)$ in Theorem 6 is not commutative, choose an element $e$ which does not commute with each element of $Q$. Then, the quantum quasigroup $\left(Q, \nabla_{1}, \Delta_{1}\right)$ will not be cocommutative. This immediately distinguishes it from the cocommutative quantum quasigroups $(A, \nabla, \Delta)$ that are provided by Theorem 5, even though those quantum quasigroups also have a commutative Moufang loop multiplication.

(b) The left composite morphism of $\left(Q, \nabla_{2}, \Delta_{2}\right)$ is

$$
x \otimes y \mapsto x \otimes x y,
$$

while the left composite morphism of $\left(Q, \nabla_{1}, \Delta_{1}\right)$ is

$$
x \otimes y \mapsto x e \otimes x \cdot y L(e)^{-1} .
$$

These automorphisms of $Q \otimes Q$ give two distinct solutions of the $Q Y B E$ in (Set, $\times, \top$ ).

Conflicts of Interest: The author declares no conflict of interest.

\section{References}

1. Benkart, G.; Madariaga, S.; Pérez-Izquierdo, J.M. Hopf algebras with triality. Trans. Am. Math. Soc. 2012, 365, 1001-1023.

2. Klim, J.; Majid, S. Hopf quasigroups and the algebraic 7-sphere. J. Algebra 2010, 323, 3067-3110.

3. Klim, J.; Majid, S. Bicrossproduct Hopf quasigroups. Comment. Math. Univ. Carolin. 2010, 51, 287-304.

4. Pérez-Izquierdo, J.M. Algebras, hyperalgebras, nonassociative bialgebras and loops. Adv. Math. 2007, 208, 834-876.

5. Smith, J.D.H. Quantum quasigroups and loops. J. Algebra 2016, 456, 46-75.

6. Smith, J.D.H. One-sided quantum quasigroups and loops. Demonstr. Math. 2015, 48, 620-636.

7. Chari, V.; Pressley, A. A Guide to Quantum Groups; Cambridge University Press: Cambridge, UK, 1994.

8. Drinfeld, V.G. On some unsolved problems in quantum group theory. In Quantum Groups; Kulish, P.P., Ed.; Springer: Berlin, Germany, 1992; Volume 1510, pp. 1-8.

9. Smith, J.D.H. Belousov's Theorem and the quantum Yang-Baxter equation. Bul. Acad. Sţiinţe Repub. Mold. Mat. 2016, 80, 7-23. 
10. Smith, J.D.H. Quantum idempotence, distributivity, and the Yang-Baxter equation. Comment. Math. Univ. Carol. 2016, accepted.

11. Smith, J.D.H.; Romanowska, A.B. Post-Modern Algebra; Wiley: New York, NY, USA, 1999.

12. Bruck, R.H. A Survey of Binary Systems; Springer: Berlin, Germany, 1971.

13. Smith, J.D.H. An Introduction to Quasigroups and Their Representations; Chapman and Hall/CRC: Boca Raton, FL, USA, 2007.

14. Elhamdadi, M.; Nelson, S. Quandles; American Mathematical Society: Providence, RI, USA, 2015.

15. Crans, A.S. Lie 2-Algebras. Ph.D. Thesis, University of California Riverside, Riverside, CA, USA, 2004.

16. Hofmann, K.H.; Strambach, K. Idempotent multiplications on surfaces and aspherical spaces. Rocky Mt. J. Math. 1991, 21, 1279-1315.

17. Albert, A.A. Non-associative algebras I. Fundamental concepts and isotopy. Ann. Math. 1942, 43, 685-707.

18. Albert, A.A. Quasigroups I. Fundamental concepts and isotopy. Trans. Am. Math. Soc. 1943, 54, 507-519.

19. Smith, J.D.H. Exterior algebra representations of commutative Moufang loops. Arch. Math. 1980, 34, $393-398$.

20. Manin, Y.I. Cubic Forms: Algebra, Geometry, Arithmetic; Nauka, USSR: Moscow, Russian, 1972.

21. Bruck, R.H. Contributions to the theory of loops. Trans. Am. Math. Soc. 1946, 60, 245-354.

22. Belousov, V.D. On the structure of distributive quasigroups (Russian). Mat. Sbornik 1960, 50, 267-298.

23. Belousov, V.D. Foundations of the Theory of Quasigroups and Loops; Nauka, USSR: Moscow, Russian, 1967.

(C) 2016 by the author; licensee MDPI, Basel, Switzerland. This article is an open access article distributed under the terms and conditions of the Creative Commons Attribution (CC-BY) license (http:/ / creativecommons.org/licenses/by/4.0/). 